\title{
DIFICULTADES DE APRENDIZAJE EN ESTUDIANTES UNIVERSITARIOS DE PRIMER AÑO ${ }^{1}$
}

\author{
LEARNING DIFFICULTIES IN FIRST YEAR \\ UNIVERSITY STUDENTS
}

\begin{abstract}
$M^{A}$ VICTORIA PÉREZ V. ${ }^{2}$, MARCO VALENZUELA CASTELLANOS ${ }^{3}$, ALEJANDRO DÍAZ M. ${ }^{4}$, JULIO ANTONIO GONZÁLEZ-PIENDA ${ }^{5}$, JOSÉ CARLOS NÚNẼÉ
\end{abstract}

\section{RESUMEN}

Los estudiantes exitosos se caracterizan por poseer estrategias de autorregulación y adoptar un enfoque profundo de aprendizaje. El desarrollo de tales habilidades se hace especialmente notorio en el primer año de estudios universitarios. En el presente trabajo se examina las relaciones entre estos aspectos en la situación de estudiantes de primer año de diversas carreras de la Universidad de Concepción, Concepción, Chile. Los resultados muestran importantes vínculos entre la adopción de un enfoque de aprendizaje profundo y el empleo de estrategias de autorregulación del aprendizaje. Se discute la conveniencia de enseñar estrategias de autorregulación en el contexto propio del currículum.

Palabras clave: Aprendizaje, estrategia, autorregulación, enfoque, estudiante.

\section{ABSTRACT}

First year at university challenges students in developing autonomous skills of learning behavior to attain successful academic outcomes. Successful students are recognized

${ }^{1}$ Esta investigación se enmarca en el Proyecto FONDECYT 1080240, denominado "Valoración de un programa de docencia para facilitar el aprendizaje activo y autorregulado”. E-mail: marperez@udec.cl

${ }^{2}$ Doctora en Psicología, Facultad de Ciencias Sociales, Universidad de Concepción. Concepción, Chile.

${ }^{3}$ Magíster en Psicología, Facultad de Ciencias Sociales, Universidad de Concepción. Concepción, Chile.

${ }^{4}$ Doctor en Psicología, Facultad de Ciencias Sociales, Universidad de Concepción. Concepción, Chile.

${ }^{5}$ Doctor en Psicología, Facultad de Psicología, Universidad de Oviedo. Oviedo, España.

${ }^{6}$ Doctor en Psicología, Facultad de Psicología, Universidad de Oviedo. Oviedo, España. 
by their self-regulated learning strategies and by the adoption of a deep approach to learning. Relations between these variables are examined in first year students of eight courses at Universidad de Concepción, Province of Concepción, Chile. Outcomes show a significant relation between these variables. Implications and projections in search of self-regulated learning strategies fostering programs are discussed.

Keywords: Learning, strategy, self-regulation, approach, student.

Recibido: 22.04.12. Aceptado: 29.09.13.

\section{ESTUDIANTES AUTORREGULADOS}

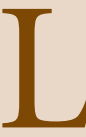

OS ACELERADOS CAMBIOs tecnológicos y sociales de comienzos del siglo XXI, junto al surgimiento de la sociedad del conocimiento demanda que el sistema educativo prepare a la persona para afrontarlos en forma autónoma, en un proceso permanente de formación que exige la capacidad de regular por sí mismo las motivaciones y las acciones (Núñez, Solano, González-Pienda y Rosário, 2006a; Solar, 1998). Así, el desarrollo de capacidades de planificación, autoevaluación del estudio y de sus resultados (Alonso, 1998) asoma como una necesidad fundamental para el logro exitoso de aprendizajes en escenarios de permanente crecimiento y modificación del conocimiento.

El estudio de los factores que distingue a los estudiantes universitarios que poseen éxito ha permitido concluir que éstos se identifican por sus capacidades de autorregulación, las que, junto a sus habilidades, posibilitan controlar y ajustar sus conductas de aprendizaje (Rosário, Núñez, González-Pienda, Almeida, Soares y Rubio, 2005b; Zimmerman, 2002). El fracaso académico se encuentra asociado, entre otras causas, a la falta de habilidad de los alumnos para controlar su comportamiento de estudio (Pérez y Díaz, 2008; Pérez, Díaz-Mujica, González-Pienda y Núñez, 2011). Por otro lado, existiría una estrecha relación entre la utilización de estrategias de autorregulación del aprendizaje, el enfoque de aprendizaje profundo y las metas de aprendizaje académico (Ruban y Reis, 2006).

La comprensión de estos aspectos contribuye a explicar los niveles de fracaso y abandono en los estudiantes universitarios de primer año mediante conceptos adicionales a los centrados en diferencias socioeconómicas (Centro de Microdatos del Departamento de Economía de la Universidad de Chile, 2008). En vistas a sus aplicaciones, permite precisar las debilidades y fortalezas con que los estudiantes emprenden el desafío 
universitario, adquiriendo relevancia el diseño de acciones de fortalecimiento de competencias de estudio autorregulado (Beneitone, Esquetini, González, Marty, Siufi y Wagenaar, 2007; Echevarría, 2003). Las estrategias de autorregulación del aprendizaje en sus dimensiones de disposición al aprendizaje (planificación de objetivos de aprendizaje, gestión del tiempo de estudio), cognitivas y metacognitivas (determinación de objetivos, metacomprensión y monitoreo) pueden ser desarrolladas a través del modelado o de la enseñanza directa mediante programas de inclusión curricular (Pérez y Díaz, 2008; Rosário, Mourão, Núñez, González-Pienda, Solano y Valle, 2007).

\section{EL DESAFÍO DE LA VIDA UNIVERSITARIA}

El paso de la enseñanza media a la enseñanza universitaria puede significar una vida nueva en otro lugar, alejarse de familia y amigos, asistir a clases y distribuir tiempo y recursos materiales en forma eficiente. Entre ambos niveles de enseñanza existe una gran diferencia de exigencias: en la Universidad aparece falta de controles para asistir a clases y tener los apuntes al día, en cambio se presentan oportunidades recreativas, culturales y de ocio, especialmente al ingreso (época de fiestas, rituales de iniciación, convivencias de camaradería, etc.). Sin embargo, se piden resultados del proceso de estudio con un mayor grado de exigencia y con menor contacto personal docente-estudiante, lo que puede afectar la motivación por el estudio, ya que ésta disminuye significativamente cuando los alumnos se ven enfrentados a una variedad abrumadora de tareas, problemas, ejercicios no significativos, evaluaciones sin opción a mejorar y a la posibilidad de tomar decisiones con autonomía y sin apoyo adecuado (Blumenfeld, 1992, Abello, Díaz, Pérez, Almeida, Lagos, González, y Strickland, 2012)). La nueva situación demanda ser capaz de actuar con mayor autorregulación y aprender a compatibilizar las exigencias académicas de la carrera con las nuevas condiciones de autonomía.

\section{ASPECTOS MOTIVACIONALES}

Para responder a las exigencias en los primeros años de estudio en la universidad, un estudiante necesita ejercitar un conjunto de comportamientos, 
procedimientos o estrategias de aprendizaje que le permitan conocer, comprender y transferir nuevos contenidos. El abordaje de cualquier actividad no se hace en blanco sino cargado de recuerdos, expectativas, habilidades, intereses, actitudes y características personales (Monereo, Castelló, Clariana, Palma y Pérez, 1994). Las creencias respecto a las propias capacidades y el juicio acerca de la capacidad para resolver una tarea (autoeficacia) determinan la orientación de un estudiante hacia un tipo de meta (Dweck, 1986; Zimmerman, Kitsantas y Campillo, 2005). Estas creencias influyen la elección y la iniciación de actividades, el esfuerzo, la constancia en el esfuerzo, la persistencia ante los obstáculos y la implicación cognitiva y afectiva en las tareas de aprendizaje (Pintrich y De Groot, 1990; Pokay y Blumenfeld, 1990).

La motivación por aprender depende tanto de los objetivos que se establecen, como de lo que se piensa al realizar una tarea (Zimmerman et al., 2005). Los estudiantes que poseen una historia académica positiva, que creen en sí mismos, con la práctica desarrollan un sentido creciente de competencia. Para que un estudiante tenga éxito académico, entendido éste como comprometerse en una actividad, mantener interés, sostener conductas de esfuerzo, persistencia en el trabajo y avanzar en el logro de sus metas, no sólo requiere disponer de potencial intelectual, sino haber desarrollado competencias para el trabajo académico unidas a un sistema de creencias orientadas al logro (Hayamizu y Weiner, 1991; Monteil, Brunot y Huguet, 1996).

La competencia en un área del conocimiento requiere tanto de la habilidad en ese ámbito como poseer un autoconcepto positivo para dicha habilidad, éste facilita la actuación en aquellas áreas valoradas como importantes para la propia definición personal. La autoestima, entendida como un sentimiento de aceptación de la persona hacia sí misma, juega un rol crítico en la vida psicológica de un estudiante (Brown y Mankowsky, 1993).

Las atribuciones causales del éxito académico, no sólo a la capacidad sino al empeño y energía puestos en la propia acción, parecen ser sus elementos claves. Es así como los estudiantes que exhiben altos logros son conscientes de los objetivos que persiguen y sus metas pueden surgir de distintas fuentes: orientación de dominio, orientación al resultado y orientación a la valoración social. Los estudiantes que tienen múltiples metas poseen una mayor capacidad de adaptarse al ambiente académico (González Cabanach, Valle, Piñeiro, Rodríguez y Núñez, 1999).

En definitiva, las actitudes de la persona ante a las tareas académicas, al despliegue de esfuerzo y energía, el modo en que se piensa a sí misma como 
aprendiz, y sus creencias sobre sus capacidades para aprender influyen en forma decisiva sobre su desempeño académico. Desarrollar la habilidad de aprender a adquirir conocimientos y utilizarlos, siendo consciente de ello, es una tarea que comienza temprano en la vida estudiantil y, al llegar a la universidad, se esperaría que el individuo posea los recursos cognitivos y conocimientos previos que le permitan entender conceptos y desarrollar tareas complejas.

\section{ESTRATEGIAS DE APRENDIZAJE}

Las estrategias de aprendizaje se entienden como un conjunto de procesos que pueden facilitar la adquisición, almacenamiento y utilización de la información (Valle, Barca, González Cabanach y Núñez, 1995). Más específicamente, son procesos de toma de decisiones (conscientes e intencionales) mediante los cuales el alumno elige y recupera los conocimientos que necesita para satisfacer una determinada demanda o lograr un objetivo, dependiendo de las características de la situación educativa en que se produce la acción (González-Pienda, Núñez, Álvarez, González-Pumariega y Roces, 1999; Monereo et al., 1994; Valle et al., 1995). Siendo actividades u operaciones mentales, su carácter intencionado demanda un plan de acción (Beltrán, 1993), así, la persona planifica y organiza su tiempo y forma de estudio.

\section{FOMENTO DE LOS PROCESOS DE AUTORREGULACIÓN}

Cuando los alumnos no cuentan con un entrenamiento autorregulatorio explícito del estudio, frecuentemente desarrollan técnicas idiosincrásicas para efectuar su trabajo académico (Zimmerman y Martínez-Pons, 1990). En la realización de tareas y exámenes muchos tienden a efectuar estimaciones inadecuadas del proceso de preparación y del proceso de ejecución de los ejercicios, por ejemplo, no releyendo con suficiente cuidado las instrucciones o el enunciado del problema, omitiendo preguntas conocidas (Ghatala, Levin, Foorman y Pressley, 1989), teniendo como consecuencia resultados bajos y desproporcionados al esfuerzo invertido.

Los alumnos cuyos profesores les enseñan y modelan de una forma sistemática estrategias de autorregulación de aprendizaje aplicadas a diferentes tareas escolares ejercitan más automáticamente su utilización (Ablard y 
Lipschultz, 1998). Para entrenar la autorregulación del aprendizaje existe una variedad de formas que deriva de distintos enfoques teóricos (Boekaerts, 1995, 1996; Boekaerts y Niemvirta, 2000; Corno, 1993; Pintrich, 2000; Pintrich y De Groot, 1990; Rosário, Mourao, Trigo, Núñez y González-Pienda, 2005a; Zimmerman, 2002; Zimmerman y Martínez-Pons, 1988). A pesar de sus diferencias, todos los modelos respaldan el planteamiento básico: los alumnos y alumnas pueden regular activamente su motivación, cognición y comportamiento $\mathrm{y}$, a través de estos procesos autorregulatorios, alcanzar sus objetivos incrementando su desempeño académico (Dembo y Eaton, 2000; Zimmerman, 1998).

\section{ESTRATEGIAS Y ENFOQUES DE APRENDIZAJE}

Existe una gran diversidad de categorías de estrategias de aprendizaje, a continuación se menciona la clasificación citada más frecuentemente (Pérez et al., 2011):

1. Las estrategias de apoyo contribuyen a generar una disposición motivacional, actitudinal y emocional adecuada para que la resolución de la tarea se lleve a buen término (Valle, González Cabanach, González, Martínez, Fernández y Pérez, 1997). Las motivacionales permiten mantener un nivel de interés necesario para emprender y mantenerse en la tarea. Las actitudinales favorecen disposiciones positivas hacia la actividad de aprendizaje; incluyen tres ámbitos de acción: sentimiento de seguridad y satisfacción personal, clima de aprendizaje e implicación en tareas escolares. Las estrategias relacionadas con la emoción permiten lograr un estado afectivo adecuado durante las tareas de aprendizaje, especialmente regulando la ansiedad.

2. Las estrategias cognitivas están directamente relacionadas con la calidad del aprendizaje (González-Pienda et al., 1999), se componen de procesos específicos para cada tarea, relacionados con conocimientos y habilidades precisas (Valle et al., 1997). Las más importantes son: repetición, selección, organización y elaboración, estas dos últimas hacen referencia a formas de procesamiento profundo, implican elaboración, reestructuración y restablecimiento de vínculos entre el aprendizaje previo y el nuevo aprendizaje (Martínez, 1998). 
3. Las estrategias metacognitivas son procedimientos de planificación, supervisión y evaluación de acciones cognitivas, conocen y regulan los procesos mentales. Son más generales que las anteriores, menos susceptibles de ser enseñadas y están estrechamente relacionadas con el conocimiento supracognitivo (Valle et al., 1997). Cuando un estudiante se encuentra ante una tarea de aprendizaje, su metacognición le permite darse cuenta de lo que sabe sobre la tarea, cuál es la naturaleza y grado de dificultad, decidir cuál es la estrategia más adecuada para resolverla y el ambiente más favorable para enfrentarse a ella (Beltrán, 1993).

4. Los enfoques de aprendizaje designan las formas en que un estudiante se dispone u orienta para desarrollar las tareas de aprendizaje (Valle, González Cabanach, Núñez, Suárez, Piñeiro y Rodríguez, 2000), distinguiéndose claramente dos tipos, uno hacia la reproducción (enfoque de orientación superficial) y otro hacia la comprensión (enfoque de orientación al significado), que sugieren que los estudiantes que autorregulan su aprendizaje poseen un enfoque de aprendizaje profundo, dirigido a la comprensión o dominio del conocimiento (Barca, Peralbo y Brenlla, 2004; Biggs, Kember y Leung, 2001; Rosário et al., 2007).

El enfoque superficial, por estar dirigido sólo al cumplimiento mínimo de una tarea, no culminaría en resultados de aprendizaje de alta calidad, ya que pondría en marcha procesos cognitivos dirigidos a un aprendizaje mecánico y memorístico para responder únicamente en un momento específico. Por su parte, el enfoque profundo se caracteriza por un alto interés y un alto grado de implicación en lo que se está aprendiendo, permitiría así descubrir el significado de lo que se va a aprender y establecer relaciones con conocimientos previos relevantes (Rosário et al., 2007).

Se asume que la persona inicia el estudio de las asignaturas con un determinado enfoque (De la Fuente, Pichardo, Justicia y Berbén, 2008) que puede variar debido al contexto de aprendizaje (Heikkilä y Lonka, 2006; Rosário et al., 2005b). Así, la percepción de los criterios de evaluación, del tipo de contenidos, del estilo de enseñanza y de las características de la tarea tendría incidencia en la adopción de uno u otro enfoque de aprendizaje por parte del alumno(a) (Valle, González Cabanach, Núñez y González-Pienda, 1998). 


\section{ESTUDIO EMPÍRICO}

En el presente trabajo, para analizar y comprender mejor la relación entre los aspectos mencionados, se hipotetiza que en los estudiantes de primer año de carreras universitarias se presentan relaciones directas y significativas entre enfoque de aprendizaje profundo, a) estrategias de disposición al aprendizaje, b) estrategias cognitivas de aprendizaje y c) estrategias metacognitivas.

Consecuentemente, se efectuó el siguiente estudio empírico con un diseño tipo descriptivo correlacional. Los participantes del estudio fueron 344 estudiantes de primer año de ocho carreras de diversas facultades (Ciencias Económicas, Ingeniería, Farmacia y Ciencias Químicas) de la Universidad de Concepción en la Provincia de Concepción, Chile. Todos proceden directamente de la Enseñanza Media, cursando por primera vez estudios universitarios (no son repitentes, ni han hecho cambio de carrera). Del total, $57 \%$ corresponde a sexo masculino y $43 \%$ a sexo femenino. Sus edades fluctuaron entre los 17 y los 23 años (promedio 18,6 años). El criterio de selección fue de tipo intencional en función de carreras con puntajes de corte al ingreso de entre 600 y 650 puntos promedio ponderados en la prueba ingreso a la universidad y una ponderación de entre 40 y $45 \%$ para la parte matemática de la prueba, asegurando así alguna homogeneidad en las características de los participantes.

Las mediciones fueron efectuadas con las escalas IPAA-Univ.; IPE Univ.; Aratex y CEA (Núñez, Solano, González-Pienda y Rosário, 2006b, Rosário et al., 2007). El instrumento está compuesto por 57 afirmaciones referidas a las estrategias de autorregulación del aprendizaje y a los enfoques de aprendizaje superficial y profundo.

Ejemplos de afirmaciones sobre Estrategias de Autorregulación del Aprendizaje:

a) Estrategias de disposición al aprendizaje: 1) Hago un plan antes de comenzar a hacer un trabajo escrito. Pienso lo que voy a hacer y lo que necesito para conseguirlo. 2) Antes de comenzar a estudiar, compruebo si tengo todo lo que necesito, para no estar siempre interrumpiendo mi estudio). 3) Antes de empezar a estudiar, me planifico el tiempo que puedo necesitar 
para comprender y aprender el tema, y cómo voy a distribuirlo entre las distintas actividades que tengo que realizar. 4) Antes de empezar a estudiar, si considero que la tarea me va a resultar aburrida, me animo a mí mismo diciéndome que podré hacer algo que me guste cuando termine.

b) Estrategias cognitivas: 1) Con el fin de comprender lo que estoy estudiando, intento descubrir ideas principales del texto. 2) Cuando estudio, intento comprender las materias, tomar apuntes, hacer resúmenes, resolver ejercicios, hacer preguntas sobre los contenidos.

c) Estrategias metacognitivas: 1) Mientras estoy estudiando, considero si mi planificación del tiempo fue correcta, o si tengo que modificarla (porque voy a necesitar más tiempo, porque me va a sobrar, etc. 2) Guardo y analizo las correcciones de los trabajos escritos o pruebas parciales, para ver dónde me equivoqué y saber qué tengo que cambiar para mejorar. 3) Mientras estoy intentando comprender, si no puedo extraer la idea de una oración importante, hago distintas actividades para aclarar su significado.

Los enfoques de aprendizaje consideran dos formas:

a) Enfoque superficial: 1) Pido a los profesores que me digan exactamente la materia que entra en el examen parcial/final, porque sólo estudio eso.

b) Enfoque profundo: 2) Estudio por la satisfacción que me produce comprender las respuestas a los "porqué".

El instrumento considera, además, información sobre horas de estudio durante la semana y fines de semana. Los datos fueron recogidos en las aulas habituales mediante la aplicación del cuestionario, requiriendo un tiempo mínimo de 20 minutos y máximo de 30 . Los estudiantes participaron voluntariamente.

Los resultados (Tabla 1) permiten confirmar la existencia de relaciones directas y significativas entre la adopción de un enfoque profundo de aprendizaje y la utilización de estrategias en las tres dimensiones del proceso de autorregulación del aprendizaje: (a) de disposición al aprendizaje, (b) estrategias cognitivas y (c) estrategias metacognitivas. 
Tabla 1. Correlación entre Enfoques de Aprendizaje y Estrategias de Autorregulación del Aprendizaje.

\begin{tabular}{|c|c|c|c|c|c|}
\hline & $\begin{array}{l}\text { Enfoque } \\
\text { Profundo }\end{array}$ & $\begin{array}{l}\text { Disposición } \\
\text { aprendizaje }\end{array}$ & $\begin{array}{l}\text { Estrategias } \\
\text { cognitivas }\end{array}$ & $\begin{array}{c}\text { Estrategias } \\
\text { metacognitivas }\end{array}$ & $\begin{array}{c}\text { Horas de } \\
\text { estudio en la } \\
\text { semana }\end{array}$ \\
\hline \multicolumn{6}{|l|}{ Disposición al } \\
\hline Aprendizaje &, $46(* *)$ & & & & \\
\hline \multicolumn{6}{|l|}{ Estrategias } \\
\hline Cognitivas &, $39(* *)$ &, $37(* *)$ & & & \\
\hline \multicolumn{6}{|l|}{ Estrategias } \\
\hline Metacognitivas &, $61(* *)$ &, $70(* *)$ &, $36(* *)$ & & \\
\hline \multicolumn{6}{|l|}{$\begin{array}{l}\text { Horas de } \\
\text { estudio en la }\end{array}$} \\
\hline $\begin{array}{l}\text { semana } \\
\text { Horas de } \\
\text { estudio en fines }\end{array}$ &, $33(* *)$ &, $36(* *)$ &, $13\left({ }^{*}\right)$ &, $31(* *)$ & \\
\hline de semana &, $26(* *)$ &, $35(* *)$ &, $12\left(^{*}\right)$ &, $25\left({ }^{* *}\right)$ &, $68\left(^{\star *}\right)$ \\
\hline
\end{tabular}

${ }^{*}$ La correlación es significante al nivel 0,05.

** La correlación es significativa al nivel 0,01 .

El enfoque profundo empleado por los estudiantes se relaciona en forma moderada con el empleo de estrategias de disposición al aprendizaje y con las cognitivas, y en forma elevada con las estrategias metacognitivas.

Los estudiantes que utilizan en forma predominante un enfoque profundo ocupan también estrategias más elaboradas de carácter metacognitivo, manifestando una mayor evaluación y toma de conciencia de sus procesos de estudio y de los resultados obtenidos, orientando su atención hacia la comprensión de lo que estudian, asumiendo en mayor medida su propia responsabilidad y mostrando mayor motivación por aprender.

Al considerar las horas de estudio durante la semana y fines de semana como indicadores del uso de enfoques de aprendizaje y de estrategias de autorregulación, las relaciones se presentan en forma importante entre el enfoque profundo y las horas dedicadas a estudiar durante la semana, y en grado menor con las horas destinadas para estudiar el fin de semana.

Se puede afirmar, entonces, en concordancia con investigaciones anteriores (Barca et al., 2004; Biggs et al., 2001; De la Fuente et al., 2008; Rosário et al., 2007), que la adopción por los estudiantes universitarios de un enfoque de aprendizaje profundo presenta relaciones directas con la utilización de estrategias de autorregulación del aprendizaje. 
Los niveles de correlación entre enfoque profundo y horas de estudio permiten afirmar que, si bien los estudiantes tienen un conocimiento de las estrategias de autorregulación, no lo convierten en acción; aunque los estudiantes saben lo que deben hacer, no saben cómo hacerlo.

Estas vinculaciones podrían explicarse por la falta de entrenamiento en estrategias de aprendizaje de estos alumnos en etapas anteriores del sistema educativo. Es posible que el paso desde un espacio educativo protegido, centrado en la adquisición de contenidos más que en la construcción autónoma de conocimiento, como ocurre en la educación chilena, sea un elemento determinante de estos resultados.

Así se pone de manifiesto la relevancia de diseñar y desarrollar acciones que permitan mejorar los procedimientos de estudio de los estudiantes universitarios. Se trata de aprendizaje de competencias instrumentales sobre aplicación y puesta en práctica habitual de procedimientos de aplicación de estrategias, más que de un conocimiento declarativo sobre ellas.

\section{DISCUSIÓN}

A partir de los productos de esta investigación se puede señalar que la muy alta relación entre las estrategias de disposición y las estrategias metacognitivas confirma el estrecho vínculo entre la fase previa o de planificación con la fase de evaluación; sin embargo, la menor relación encontrada entre estas dos dimensiones con las estrategias cognitivas, propias de la fase de ejecución, muestra la necesidad de implementar programas de apoyo y enseñanza de competencias en estrategias de autorregulación a los alumnos de primer año, tomando en consideración que estas estrategias pueden ser enseñadas y aprendidas (Núñez et al., 2006a; Rosário et al., 2007). El disponer de esta información permitirá indagar qué condiciones provocan estos resultados $y$, por otra parte, iniciar acciones para el fortalecimiento de sus condiciones ventajosas y de mejoramiento de aspectos deficitarios.

La autorregulación y la actividad de estudio son competencias que deberían aplicar y desarrollar los alumnos en la universidad (Allgood, Risko, Álvarez y Fairbanks, 2000), y que les permitirán ser autónomos al aprender activa y significativamente durante toda su vida, como se plantea en la Declaración de Bolonia (González y Wagenaar, 2003).

Parece recomendable incorporar la enseñanza de las estrategias de aprendizaje en los programas de formación de los profesores para que, posteriormente, puedan ser incluidas, como método para estudiar y no como 
contenido, en los respectivos currículos de áreas de conocimiento (Hadwin y Winnie, 1996; Hattie, Biggs y Purdie, 1996; Simpson, Hynd, Nist y Burrel, 1997). Los entrenamientos en estrategias de autorregulación realizados por los propios docentes, en contextos, tareas y contenidos propios del currículum, incrementan la probabilidad de transferencia de esos aprendizajes (Salomon y Perkins, 1989; Simpson et al., 1997; Zimmerman, Bonner y Kovach, 1996).

En el desarrollo de las tareas propias del currículum de su disciplina, los profesores pueden ofrecer modelos a sus alumnos y discutir con ellos la aplicación de estrategias de autorregulación a situaciones cotidianas de enseñanza-aprendizaje, facilitando su aplicación supervisada y, posteriormente, autónoma. Cada comportamiento autorregulatorio, tal como el establecimiento de una meta o la autoevaluación de la conducta de estudio, puede ser enseñado directamente o modelado por profesores o compañeros.

\section{REFERENCIAS}

Abello, R., Díaz, A., Pérez, M.V., Almeida, L., Lagos, I., González, J. y Strickland B. (2012). "Vivencias e implicación académica en estudiantes universitarios: adaptación y validación de escalas para su evaluación”. Estudios Pedagógicos. XXXVIII, 2: 7-19.

Ablard, K. y Lipschultz, R. (1998). "Self-regulation in high-achieving students: relations to advanced reasoning, achievement goals, and gender". Journal of Educational Psychology 90 (1), 94-101.

Allgood, W.; Risko, V.; Âlvarez, M. y Fairbanks, M. (2000). "Factors that influence study". En R.F. Flippo y D.C. Caverly (Coord.), Handbook of college reading and study strategy research (pp. 201-219). N. J: LEA.

Alonso, M.N. (1998). "La propuesta de Reforma Educacional. '¿A qué tanta manía pedagógica?"”. Atenea 478: 31-48.

Barca, A.; Peralbo, M. y Brenlla, J. (2004). "Atribuciones causales y enfoques de aprendizaje: la escala SIACEPA". Psicothema 16 (1): 94-103.

Beltrán, J. (1993). Procesos, estrategias y técnicas de aprendizaje. España: Síntesis.

Beneitone, P.; Esquetini, C.; González, J.; Marty, M.; Siufi, G. y Wagenaar, R. (2007). Reflexiones y perspectivas de la educación superior en América Latina. Informe final -Proyecto Tuning- América Latina 2004-2007. Bilbao: Universidad de Deusto.

Biggs, J., Kember, D. y Leung, D. (2001). "The revised two-factor Study process Questionnaire: R-SPQ-2F”. British Journal of Educational Psychology 71 (1): 133-149.

Blumenfeld, P.C. (1992). "Classroom learning and motivation: Clarifying and expanding goal theory”. Journal of Educational Psychology 84 (3): 272-281. 
Boekaerts, M. (1995). "Self-regulated learning: bridging the gap between metacognitive and metamotivation theories". Educational Psychologist 30 (4): 195-200.

(1996). "Self-regulated learning at the junction of cognition and motivation". European Psychologist 1 (2): 100-112.

Boekaerts, M. y Niemvirta, M. (2000). "Self-regulated learning: finding a balance between learning goals and ego-protective goals". En M. Boekaerts, P. Pintrich \& M. Zeidner (Eds.), Handbook of Self-regulation (pp. 417-450). New York: Academic Press.

Brown, J.D. y Mankowsky, T. A. (1993). "Self-Esteem, Mood, and Self-Evaluation: Changes in Mood and the Way you see You". Journal of Personality and Social Psychology 64 (3), 421-430.

Centro de Microdatos del Departamento de Economía de la Universidad de Chile (2008). "Informe final estudio sobre causas de la deserción universitaria”. Extraído el 12 de marzo de 2009 de http://www.mece2.com/portal/ component/option,com_docman/task,doc_download/gid,409/Itemid,35/ lang,spanish/

Corno, L. (1993). "The best-laid plans: Modern conceptions of volition and educational research". Educational Researcher 22 (2): 14-22.

De la Fuente, J., Pichardo, M., Justicia, F. y Berbén, A. (2008). "Enfoques de aprendizaje, autorregulación y rendimiento en tres universidades europeas". Psicothema 20 (4): 705-711.

Dembo, M.H. y Eaton, M.J. (2000). "Self-Regulation of academic learning in middle-level schools”. Elementary School Journal 100 (5): 473-490.

Dweck, C.S. (1986). "Motivational process afecting learning". American Psychologist 41 (10), 1040-1048.

Echevarría, G. (2003). "El sistema europeo de transferencia de créditos y su implementación en universidades del MERCOSUR". Revista Calidad en la Educación 19: 197-206.

Ghatala, E.; Levin, J.; Foorman, B. y Pressley, M. (1989). "Improving children's regulation of their reading Prep time". Contemporary Educational Psychology 14 (1): 49-66.

González, J. y Wagenaar, R. (Eds.) (2003). Tuning Educational Structures in Europe. Informe final. Fase Uno. Bilbao: Universidad de Deusto.

González Cabanach, R., Valle, A., Piñeiro, I., Rodríguez, S. y Núñez, J. (1999). "El ajuste de los estudiantes con múltiples metas a variables significativas del contexto académico". Psicothema 11 (2): 313-323.

González-Pienda, J.; Núñez, J.; Álvarez, L.; González-Pumariega, S. y Roces, C. (1999). "Comprensión de problemas aritméticos en alumnos con y sin éxito". Psicothema 11 (3): 505-515.

Hadwin, A.F. y Winnie, P.H. (1996). "Study strategies have meager support. A review with recommendations for implementation”. Journal of Higher Education 67 (6): 693-715.

Hattie, J.; Biggs, J. y Purdie, N. (1996). “Effects of Learning Skills Interventions 
on Student learning: A Meta-analysis". Review of Educational Research 66 (2): 99-136.

Hayamizu, T. y Weiner, B. (1991). "A test of Dweck's model of achievement goals as related to perceptions of ability". The Journal of Experimental Education 59 (3): 226-234.

Heikkilä, A. y Lonka, K. (2006). "Studying in higher education: students' approaches to learning, self-regulation, and cognitive strategies". Studies in Higher Education 31 (1): 99-117.

Martínez, J. (1998). "Estrategias cognitivas y motivacionales en estudiantes universitarios de pedagogía". Ponencia presentada en el II Congreso Iberoamericano de Psicología. Universidad de Barcelona, España.

Monereo, C.; Castelló, M.; Clariana, M.; Palma, M. y Pérez, M.L. (1994). Estrategias de enseñanza y aprendizaje. Barcelona: Graó.

Monteil, J.M.; Brunot, S. y Huguet, P. (1996). "Cognitive performance and attention in the classroom: An interaction between past and present academic experiences”. Journal of Educational Psychology 88 (2): 242-248.

Núñez, J.; Solano, P.; González-Pienda, J. y Rosário, P. (2006a). “El aprendizaje autorregulado como medio y meta de la educación”. Papeles del Psicólogo 27 (3): 139-146.

Núñez, J.; Solano, P.; González-Pienda, J. y Rosário, P. (2006b). "Evaluación de los procesos de autorregulación mediante autoinforme”. Psicothema 18 (3): 353-358.

Pérez, M.V. y Díaz, A. (2008). "Valoración de un programa de docencia para facilitar el aprendizaje activo y autorregulado”. Proyecto FONDECYT No 1080240.

Pérez, M.V.; Díaz-Mujica, A.; González-Pienda, J.A. y Núñez, J.C. (2011). “Autorregulación del aprendizaje en educación superior”. En J. Catalán (Ed.), Psicología Educacional: proponiendo rumbos, problemáticas y aportaciones. La Serena: ULS.

Pintrich, P. (2000). "An achievement goal theory perspective on issues in motivation terminology, theory and research". Contemporary Educational Psychology 25 (1): 92-104.

Pintrich, P. y De Groot, E. (1990). "Motivational and self-regulated learning components of classroom academic performance. Special Section: Motivation and efficacy in education: Research and new directions". Journal of Educational Psychology 82 (1): 33-40.

Pokay, P. y Blumenfeld, Ph.C. (1990). "Predicting achievement early and late in the semester: The role of motivation and use of learning strategies. Special Section: Motivation and efficacy in education: Research and new directions". Journal of Educational Psychology 82 (1), 41-50.

Rosário, P.; Mourao, R.; Trigo, J.; Núñez, J. y González-Pienda, J. (2005a). “SRL Enhancing Narratives: Testas' (Mis)adventures”. Academic Exchange Quarterly 9 (4): 73-77.

Rosário, P.; Núñez, J.; González-Pienda, J.; Almeida, L.; Soares, S. y Rubio, M. 
(2005b). "El aprendizaje escolar examinado desde la perspectiva del 'Modelo 3P' de J. Biggs”. Psicothema 17 (1): 20-30.

Rosário, P.; Mourão, J.; Núñez, J.; González-Pienda, J.; Solano, P. y Valle, A. (2007). Eficacia de un programa instruccional para la mejora de procesos y estrategias de aprendizaje en la enseñanza superior. Psicothema, 19 (3), 422-427.

Ruban, L. y Reis, S. (2006). Patterns of self-regulatory strategy among lowachieving and high achieving university students. Roeper Review, 28 (3), 148-156.

Salomon, G. y Perkins, D. (1989). Rocky roads to transfer: rethinking mechanisms of a neglected phenomenon. Educational Psychologist, 24 (2), 113142.

Simpson, M.; Hynd, C.; Nist, C. y Burrel, K. (1997). College academic assistance programs and pratices. Educational Psychology Review, 9 (1), 39-87.

Solar, M.I. (1998). La Reforma Educacional chilena: Problemática y desafíos. Atenea, 478, 11-29.

Valle, A.; Barca, A.; González Cabanach, R. y Núñez, J. (1995). Las estrategias de aprendizaje: Una aproximación teórica y conceptual. Revista Galega de Psicopedagoxia, 8, 30-57.

Valle, A.; González Cabanach, R.; González, R., Martínez; S., Fernández, M. y Pérez, J. (1997). Orientaciones Motivacionales en estudiantes universitarios. Actas $1^{\circ}$ Congreso Luso-español de Psicología de la Educación, 258-275.

Valle, A., González Cabanach, R., Núñez, J. y González-Pienda, J. (1998). Variables cognitivo-motivacionales, enfoques de aprendizaje y rendimiento académico. Psicothema, 10 (2), 393-412.

Valle, A.; González Cabanach, R.; Núñez, J.; Suárez, J.; Piñeiro, I. y Rodríguez, S. (2000). Enfoques de aprendizaje en estudiantes universitarios. Psicothema, 12 (3), 368-375.

Zimmerman, B.J. (1998). Developing self-fulfilling cycles of academic regulation: An analysis of exemplary instructional models. In D. H. Schunk y B.J. Zimmerman (Eds.), Self-Regulated learning. From teaching to Self-Reflective Practice (pp. 1-19). Hillsdale, N. J: Lawrence Erlbaum Associates, Inc.

(2002). Becoming a self-regulated learner: an overview. Theory Into Practice, 41 (2), 64-70.

Zimmerman, B.J. y Martinez-Pons, M. (1988). Construct validation of a strategy model of student self-regulated learning. Journal of Educational Psycho$\log y, 80$ (3), 284-290.

(1990). Student differences in self-regulated learning: Relating grade, sex, and giftedness to self-efficacy and strategy use. Special Section: Motivation and efficacy in education: Research and new directions. Journal of Educational Psychology, 82 (1), 51-59.

Zimmerman, B.J.; Bonner, S. y Kovach, R. (1996). Developing self-regulated learners: Beyond achievement to self-efficacy. Washington, DC: American Psychological Association. 
Zimmerman, B.J.; Kitsantas, A. y Campillo, M. (2005). Evaluación de la autoeficacia regulatoria: una perspectiva social cognitiva. Evaluar, 5, 1-21. 